\title{
An Warning Information System for Rice Disease Based on WebGIS
}

\author{
Xuan Xie, Si-qing Yang \\ Hunan University of Humanities, Science and Technology, Loudi, 417000, China
}

\begin{abstract}
This paper focuses on the construction and database design of the rice disease early warning information system based on WebGIS, and briefly analyzes the development software of WebGIS. On the basis of establishing database of main rice diseases, with the Internet as the system platform, using WebGIS technology, ASP network programming technology, network database technology, artificial intelligence technology and other modern information system development method, it designs plant diseases and insect pests of rice early warning information system based on WebGIS. The system realizes the rice disease information collection, data transmission, disease diagnosis, effect of real-time release.
\end{abstract}

Keywords: WebGIS; Rice disease; Warning; Database

\section{Introduction}

Rice pests and diseases are one of the important factors affecting grain yield. It is of great significance to promptly and accurately publish information on the occurrence and change trends of rice pests and diseases, and to increase the enthusiasm of farmers and agribusiness production and promote the sustainable development of rural economy.

With the development of information technology, network geographic information system technology, network database management technology, ASP network programming technology, AI artificial intelligence technology have been applied more and more to the detection and early warning of rice pests and diseases. Based on the Internet platform and combined with big data analysis methods, a rice GIS-based early warning information system based on WebGIS was designed to translate raw data that can be obtained from actual production to reflect the changing trends of rice pests and diseases into clear and intuitive maps and statistical reports., It is convenient for users to inquire [1]. Through forecasting and analysis, using charts to show the degree of occurrence of pests and areas of change, is simple and clear, to achieve the function of early warning and forecast of rice pests and diseases, and has a certain practical value and high economic benefits for the harvest of crop yields.

\section{System composition}

System components are divided into four modules, namely, rice pest and disease data management, rice pest and disease monitoring data, warning information release, and comprehensive information management [2], see Figure 1. 


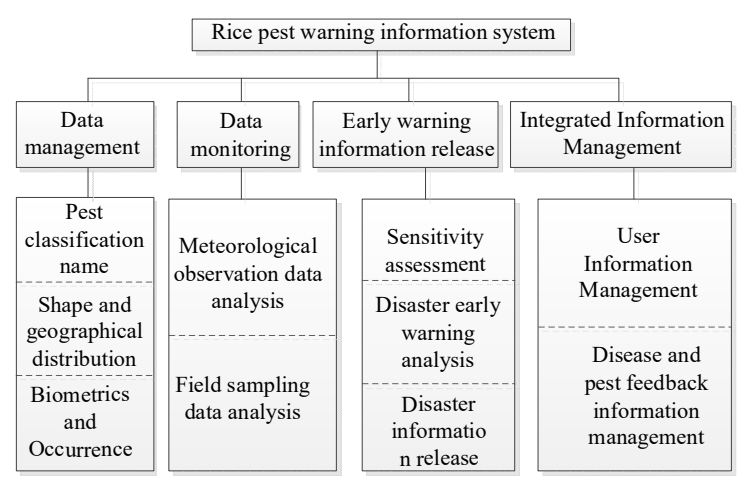

Fig. 1 System block diagram

The pest and disease data management module mainly manages the basic data from the national rice pest and disease database. China has a vast territory, with a wide range of climatic conditions in different regions. The timing of the occurrence of rice pests and diseases, the geographical location of occurrence, the occurrence of pests and diseases, and the types of pests and diseases vary. Therefore, methods for the classification and management of diseases and pests, and integrated prevention and control strategies Must be treated differently. The pest and disease monitoring data module mainly analyzes and processes the temperature, rainfall, sunshine intensity, and wind direction wind speed provided by the meteorological observing station, as well as field sampling data provided by plant protection stations. Data analysis and processing can call the function of data analysis and processing in Excel, and display the development trend of pests and diseases through the establishment of line charts with data markers. The core of the whole system is the early warning information release module, which conducts early warning and analysis based on historical pest and disease information and on-site collected pest information to accurately predict the probability of future occurrence of pests and diseases and development areas, thus providing decision-making advice on pest control. It includes such contents as sensitivity assessment, disaster early warning analysis, and disaster information release. The integrated information management module manages user information of the entire system on the one hand, completes user registration, login, and information modification, and on the other hand also receives user feedback information.

\section{Database design}

Due to the large amount of data that needs to be processed in the rice pest warning information system, the normal database cannot meet this requirement. The system uses an open source database. Through the optimization of the data structure model, the spatial data and attribute data are stored and managed in a unified manner, and a reasonable spatial database and attribute database are established. The structure of the database is shown in Figure 2.

\section{Analyze and Process Data}

The data that needs to be analyzed and processed by the rice pest warning information system comes from basic geographic information data, pest and disease monitoring data, and meteorological data. Basic geographic information data can be downloaded through the National Basic Geographic Information Network, and vectorization of administrative maps 
and regional hydrogeological maps can be obtained. The pest and disease monitoring data includes disease and insect habits, field sampling data, remote sensing monitoring data, etc., and can be compiled based on regional pest surveys and regional historical data. Meteorological data includes weather, soil relative humidity, rainfall, sunshine hours, and wind speed.

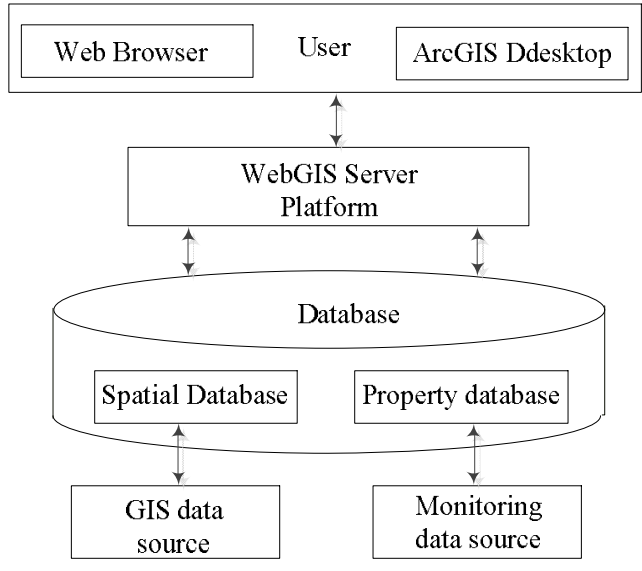

Fig. 2 Database structure block diagram

Because the data types in the database are not the same, it is necessary to further divide them into spatial data and attribute data before analyzing and processing the data. For the spatial data of rice pests and diseases, when obtaining data, even if it is the same area, the map used has different map coordinates, map units, map scales, and map projection methods. When capturing data, maps need to be projected. Transformation, converted to the same coordinate system, the same scale of data. Therefore, the graphic data must be standardized. For the attribute data, the data needs to be re-encoded and stored in the form of a table to prepare for the construction of the attribute database.

In addition, to conduct effective and integrated management of the massive data in the system, the data must be standardized and data-encoded before data organization. All data related to graphic libraries should be organized in the concept of "layers", and each layer can be linked with the property library through "unified coding" so that the graphic library and the property library can be organically combined, and the users can conveniently Call the desired frame file, edit the layer, and query the spatial information of each layer of data.

Design Space Database

Using ArcGIS Server to build a multi-user WebGIS platform to develop Web applications and services, B/S development tools built with ArcObjects can support SDE, Shape, and Arc/Info Coverage format spatial databases [3]. Geodatabase is a spatial database that uses standard relational database technology to represent geographic information. It adds pest and pest data, rainfall data and various basic geographic information data in ArcMap 10.5. Open the attribute table to enter and edit related attribute fields, and then use the ArcCatalog resource manager of geographic data to create a Personal Geodatabase.

Design Attribute Database

After designing the spatial database, the database of rice pest pests and meteorological warnings is designed. The reason is that each layer in the spatial database corresponds to 
several attribute information, and the corresponding attribute data is stored in the SQL Server database. The system creates a property table for each layer in the designed SQL Server property database and enters the relevant property data.

\section{Associated Database}

The spatial data and the attribute data corresponding to the layer are stored in the (*.dbf) table that comes with the Shape file. In addition, the attribute data is stored in the SQL Server database. The system creates a Class, New Relation-ship Class under the Personal Geodatabase, uses the "unified coding" as the key, executes Append to implement the association between the Shape Graph Database and the SQL Server property database, Can realize the integration storage of space and attribute database [4].

\section{System function implementation}

\section{Pest and Disease Data Collection and Maintenance}

Data collection is from the rice pests and diseases map taken from the field, collected information on pests and diseases, such as bacterial leaf blight picture, black stripe dwarf disease pictures, names of various pests and diseases, pest characteristics, methods of prevention and treatment for plant protection professional and technical personnel Enter the system. Data maintenance is performed by the technicians of the specialized management system. After the login, the original data in the system is modified, updated, added, deleted, sequenced, inserted, and other data maintenance work.

\section{Pest Information Inquiry and Retrieval}

The system has the query function of pest and disease information, and historical data of rice pests and diseases recorded in the property database in previous years or within a certain period of time can be found at any time. The "Alert Information List" icon provided by the system will flash in time to indicate the latest occurrence of rice pests, corresponding control technologies, and latest announcement information. By inputting the keywords to be searched, the pest information search function can be realized, and the pathogens, symptoms, predisposing factors, and prevention and control measures of rice pests and diseases can be retrieved. In the process of data entry, it has a full-screen editing function, which can easily find, replace, classify, and summarize data already entered.

\section{Regional Distribution and Early Warning of Diseases and Pests}

The function of distribution and early warning of pests and diseases is to use the pest warning information release module to extract scientific data from the property database, such as the sensitivity status of pests and diseases, and the predisposing factors of pests and diseases, and to scientifically analyze and evaluate them. The way to display the analysis results is shown in Figure 3. In functional design, we can let the system not only do automatic warning analysis, but also can do artificial warning analysis, and can also simulate rice pests and diseases that have occurred in history. Through comparative analysis results, it is convenient for system development technicians to understand what problems the system model has in practical applications and find out where improvement is needed. 


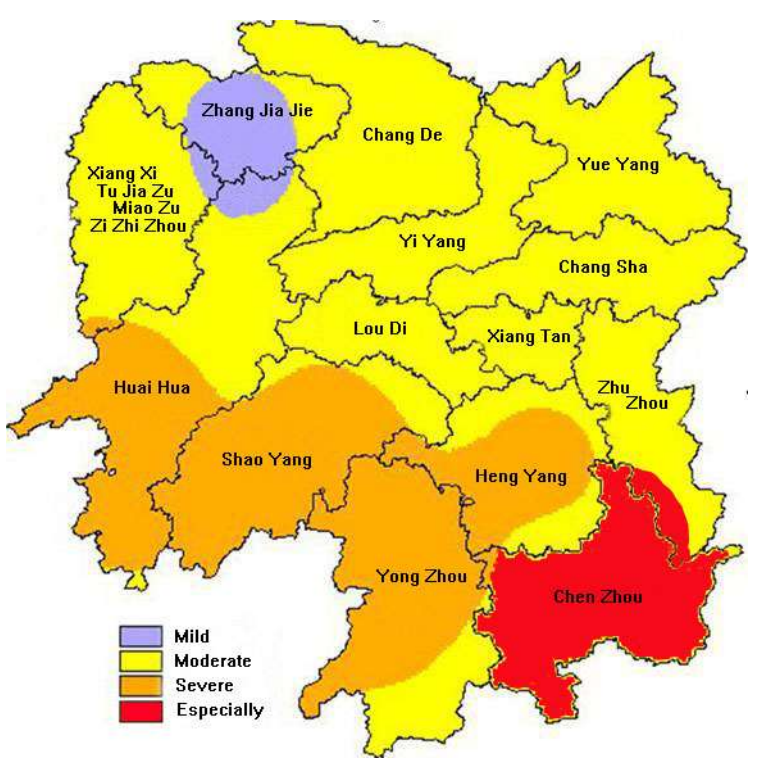

Fig. 3 Disease area map

\section{Conclusions}

This paper introduces the design and implementation of the rice pest warning information system based on WebGIS, applies the network technology and database technology to the WebGIS development platform, and designs an application system with simple operation, good security, and good early warning effect, and basically achieves the expected functions.

\section{References}

[1] Luo Qingwen, Jiang Yaopei, Lu Zhenmei, et al. Research and Application of the Rice Early Warning System for Catastrophic Diseases in Agriculture. Agricultural Network Information [J]. 2008(12):7-10.

[2] Liu Xiaohong, Dai Xiaopeng, Liu Haibo et al. WebGIS based design of early warning platform for rice pests and diseases. Agricultural disaster research [J]. 2012, 2(01): 6-8.

[3] Chen Xuerui, Jia Jingdun, Gao Wanlin et al. WebGIS-based value evaluation system for agricultural scientific and technological achievements. Journal of Agricultural Machinery [J]. 2017, 67(05): 238-244.

[4] Huang Chong, Liu Wancai, Jiang Yuying et al. Digital Monitoring and Early Warning System for Major Plant Diseases and Insect Pests. Chinese Journal of Agricultural Mechanization[J]. 2016, 48(03): 196-199. 